\title{
INTERVAL VERSIONS OF MAX-MIN METHOD FOR SOLVING THE MULTI OBJECTIVE INTERVAL TRANSPORTATION PROBLEM
}

\author{
G. SUDHA \& K. GANESAN
}

Department of Mathematics, SRM Institute of Science and Technology, Kattankulathur, Tamil Nadu, India

Received: Jun 08, 2020; Accepted: Jun 28, 2020; Published: Sep 09, 2020; Paper Id.: IJMPERDJUN20201133

\section{INTRODUCTION}

The classical problem of transport (TP) can be seen as a special case of the problem of linear programming, and its model is applied to calculate how many units of the goods output are to be transported from each origin to different destinations, meeting supply and demand constraints, thus reducing total transport costs. TP is commonly used in economic growth, industrial administration, passenger and freight modes, etc., and plays a critical role in countries ' economic development. Single objective TP is not sufficient to solve complex real-life scenarios; thus, we consider MOITP (multi-objective interval transport problem), which has been applied in many science fields, including engineering, economics and logistics; Where optimal choices are taken in the face of trade-offs between two or more competing goals Examples of multi-objective optimization problems involving two or three objectives are cost reduction and enhancement of comfort when buying a car and optimizing performance when reducing vehicle fuel consumption and pollutant emissions. In most real-life transport networks, transportation prices are charged by customers, and the sale of the goods is profited to the producer's benefit. Therefore suppliers would like to optimize the benefit while at the same time wishing customers to reduce the expense of objective functions. The parameters of the question of transport are the costs of transport, the quantity of goods available at the point of supply and the quantity required at the point of request. In earlier days, the transportation issue was based on the assumption that the parameters of supply, demand, and costs were understood accurately. But not all parameters of the transportation problem are necessarily defined exactly in real-life applications. It can have interval value. This paper will take into account considerations relating to the parameters of supply and demand in TP. In line with that viewpoint, this paper is constructed with these transport problem parameters as interval values.

\section{THE STUDY OF LITERATURE}

Several methods exist in the literature to solve these kinds of problems. Ishibuchi and Tanaka [9] proposed multiobject if programming to optimize the interval's objective function. Chanas et.al [2] studies Interval and Fuzzy extensions of classic transport problems. Das et.al [4] generated a multi-objective transport problem with 
parameters for the rate, source and destination intervals. Chinneck and Ramadan [3] discussed linear programming from intervals with coefficients. Ganesan and Veeramani [6] have proposed a new arithmetical operation of the number interval. Ganesan [7] studied at intervals certain properties of the matrices. Oliveira and Antunes [11] discussed multiple linear target programming models with interval coefficients. Pandian and Natrajan [12] have proposed a new approach to seeking an optimal solution for the transportation of integer problems at complete intervals. Sudhakar and Navaneetha Kumar [21] have developed a new approach to finding an optimal solution to problems with integral transit intervals. Fegade et.al [5] has used interval and triangular membership functions to provide an optimal solution to the problem of transport. Ramesh and Ganesan [13],[14] suggested linear interval programming with a generalized arithmetic interval, and also introduced duality theory for problems with linear interval programming. Safi and Razmjoo [16] answered the issue of interval parameters for fixed charges. Akilbasha et.al [1] dealt with a innovative and detailed approach to solving problems of fullinterval integer transport. Sophia Porchelvi and Anitha [18] suggested a comparative analysis of the optimal solution between conveyance interval and transhipment interval problem. Ramesh et.al [15] suggested a two-vehicle solution that would cost different transportation intervals - a modern approach. Gurupada Maity et.al [8] presented the multi-objective, interval-assessed, time model of sustainable development transport. Sathya Geetha and Selvakumari [19] proposed a new way of using pentagonal fuzzy numbers to solve hazy transportation problems.

In this paper we propose a max-min algorithm under a generalized arithmetic interval to solve the problem of multi-objective interval transportation without converting the crisp equivalent question. In this paper we propose a maxmin algorithm under generalized interval arithmetic to solve the multi objective interval transportation problem without converting the crisp equivalent question.

The structure of this article is given as follows: Recall related basic concepts in section. Section 3 deals with the basic definitions. Section 4 deals with the problem of multi-objective interval transportation and the related results. Section 5 introduces the max-min algorithm under generalized interval arithmetic. Section 6 provides an example in numbers to illustrate the theory developed in this paper. Section 7 concludes this paper.

\section{PRELIMINARIES}

The purpose of this segment is to provide some observations, ideas and results which are useful in our further consideration.

\section{Interval Numbers}

Let $\tilde{a}=\left[a_{1}, a_{2}\right]=\left\{x \in R: a_{1} \leq x \leq a_{2}\right.$ and $\left.a_{1}, a_{2} \in R\right\}$ be an interval on the real line $R$. If $\tilde{a}=a_{1}=a_{2}=a$, then $\tilde{\mathrm{a}}=[\mathrm{a}, \mathrm{a}]=\mathrm{a}$ is a real number (or a degenerate interval). We shall make use of the terms interval and interval number interchangeably. The mid-point and width (or half-width) of an interval number $\tilde{a}=\left[a_{1}\right.$, $\left.a_{2}\right]$ are defined as $\mathrm{m}(\tilde{\mathrm{a}})=\left(\frac{\mathrm{a}_{1}+\mathrm{a}_{2}}{2}\right)$ and $\mathrm{w}(\tilde{\mathrm{a}})=\left(\frac{\mathrm{a}_{2}-\mathrm{a}_{1}}{2}\right)$. The interval number $\tilde{\mathrm{a}}$ can also be expressed in terms of its midpoint and width as $\tilde{a}=\left[a_{1}, a_{2}\right]=\langle m(\tilde{a}), w(\tilde{a})\rangle$. We use IR to denote the set of all interval numbers defined on the real line $R$. 


\section{Ranking of Interval Numbers}

Sengupta and Pal [17] suggested a easy and powerful index to compare any two intervals on IR through the satisfaction of decision-makers .

Definition: Let $\preceq$ be an extended order relation between the interval numbers $\tilde{a}=\left[a_{1}, a_{2}\right], \tilde{b}=\left[b_{1}, b_{2}\right]$ in IR, then for $m(\tilde{a})<m(\tilde{b})$, we construct a premise $\left(\tilde{a}{ }^{\circ} \tilde{b}\right)$ which implies that $a$ is inferior to $\tilde{b}$ (or $\tilde{b}$ is superior to $\tilde{a}$ ).

An acceptability function $\mathrm{A}_{\preceq}: \operatorname{IR} \times \mathrm{IR} \rightarrow[0, \infty)$ is defined as:

$$
A_{\beth}(\tilde{a}, \tilde{b})=A(\tilde{a} \preceq \tilde{b})=\frac{m(\tilde{b})-m(\tilde{a})}{w(\tilde{b})+w(\tilde{a})}, \text { where } w(\tilde{b})+w(\tilde{a}) \neq 0 .
$$

A $\_$may be interpreted as the grade of acceptability of the first interval number to be inferior to the second interval number. For any two interval numbers $\tilde{a}$ and $\tilde{b}$ in $\operatorname{IR}$ either $\mathrm{A}(\tilde{\mathrm{a}} \preceq \tilde{\mathrm{b}}) \geq 0$ (or) $\mathrm{A}(\tilde{\mathrm{b}} \succeq \tilde{a}) \succeq 0$ (or) $\mathrm{A}(\tilde{\mathrm{a}} \preceq \tilde{\mathrm{b}})=0$ (or) $\mathrm{A}(\tilde{\mathrm{b}} \succeq \tilde{\mathrm{a}})=0$ (or) $\mathrm{A}(\tilde{\mathrm{a}} \preceq \tilde{\mathrm{b}})+\mathrm{A}(\tilde{\mathrm{b}} \preceq \tilde{\mathrm{a}})=0$. If $\mathrm{A}(\tilde{\mathrm{a}} \preceq \tilde{\mathrm{b}})=0$ and $\mathrm{A}(\tilde{\mathrm{b}} \preceq \tilde{\mathrm{a}})=0$, then we say that the interval numbers $\tilde{a}$ and $\tilde{b}$ are equivalent (non-inferior to each other) and we denote it by $\tilde{a} \approx \tilde{b}$. Also if $A(\tilde{a} \preceq \tilde{b}) \geq 0$, then $\tilde{a} \preceq \tilde{b}$ and if $A(\tilde{b} \preceq \tilde{a}) \geq 0$, then $\tilde{b} \preceq \tilde{a}$.

\section{A New Interval Arithmetic}

Ming Ma et al. [10] suggested a new fuzzy arithmetic focused on the index of locations and the index function of fuzziness. For the ordinary arithmetic the position index number is taken, while in the lattice L the fuzziness index functions are assumed to obey the lattice law which is the least upper bound and the greatest lower bound. That is for $a, b \in L$ we define $a \vee b=\max \{a, b\}$ and $a \wedge b=\min \{a, b\}$.

For any two intervals $\tilde{a}=\left[a_{1}, a_{2}\right], \tilde{b}=\left[b_{1}, b_{2}\right] \in \operatorname{IR}$ and for $* \in\{+,-, \cdot, \div\}$, the arithmetic operations on $\tilde{a}$ and $\tilde{b}$ are defined as:

$$
\tilde{a} * \tilde{b}=\left[a_{1}, a_{2}\right] *\left[b_{1}, b_{2}\right]=\langle m(\tilde{a}), w(\tilde{a})\rangle *\langle m(\tilde{b}), w(\tilde{b})\rangle=\langle m(\tilde{a}) * m(\tilde{b}), \max \{w(\tilde{a}), w(\tilde{b})\}\rangle .
$$

In particular

$$
\begin{aligned}
& \text { (i). Addition : } \tilde{a}+\tilde{b}=\langle\mathrm{m}(\tilde{\mathrm{a}}), \mathrm{w}(\tilde{\mathrm{a}})\rangle+\langle\mathrm{m}(\tilde{\mathrm{b}}), \mathrm{w}(\tilde{\mathrm{b}})\rangle=\langle\mathrm{m}(\tilde{\mathrm{a}})+\mathrm{m}(\tilde{\mathrm{b}}), \max \{\mathrm{w}(\tilde{\mathrm{a}}), \mathrm{w}(\tilde{\mathrm{b}})\}\rangle \text {. } \\
& \text { (ii). Subtraction : } \tilde{a}-\tilde{b}=\langle\mathrm{m}(\tilde{\mathrm{a}}), \mathrm{w}(\tilde{\mathrm{a}})\rangle-\langle\mathrm{m}(\tilde{\mathrm{b}}), \mathrm{w}(\tilde{\mathrm{b}})\rangle=\langle\mathrm{m}(\tilde{\mathrm{a}})-\mathrm{m}(\tilde{\mathrm{b}}), \max \{\mathrm{w}(\tilde{\mathrm{a}}), \mathrm{w}(\tilde{\mathrm{b}})\}\rangle \text {. }
\end{aligned}
$$

(iii). Multiplication : $\tilde{a} \times \tilde{b}=\langle\mathrm{m}(\tilde{\mathrm{a}}), \mathrm{w}(\tilde{\mathrm{a}})\rangle \times\langle\mathrm{m}(\tilde{\mathrm{b}}), \mathrm{w}(\tilde{\mathrm{b}})\rangle=\langle\mathrm{m}(\tilde{\mathrm{a}}) \times \mathrm{m}(\tilde{\mathrm{b}}), \max \{\mathrm{w}(\tilde{\mathrm{a}}), \mathrm{w}(\tilde{\mathrm{b}})\}\rangle$.

(iv). Division : $\tilde{a} \div \tilde{b}=\langle\mathrm{m}(\tilde{\mathrm{a}}), \mathrm{w}(\tilde{\mathrm{a}})\rangle \div\langle\mathrm{m}(\tilde{\mathrm{b}}), \mathrm{w}(\tilde{\mathrm{b}})\rangle \quad=\langle\mathrm{m}(\tilde{\mathrm{a}}) \div \mathrm{m}(\tilde{\mathrm{b}}), \max \{\mathrm{w}(\tilde{\mathrm{a}}), \mathrm{w}(\tilde{\mathrm{b}})\}\rangle$, provided $\mathrm{m}(\tilde{\mathrm{b}}) \neq \tilde{0}$. 


\section{MAIN RESULTS}

Consider a fully interval transportation problem with $\mathrm{m}$ sources and $\mathrm{n}$ destinations involving interval numbers. Let $\tilde{a}_{\mathrm{i}} \succeq \tilde{0}$ be the availability at source $\mathrm{i}$ and $\tilde{\mathrm{b}}_{\mathrm{j}}$, $\left(\tilde{\mathrm{b}}_{\mathrm{j}} \succeq \tilde{0}\right)$ be the requirement at destination $\mathrm{j}$. Let $\tilde{\mathrm{c}}_{\mathrm{ij}}\left(\tilde{\mathrm{c}}_{\mathrm{ij}} \succeq \tilde{0}\right)$ be the unit interval transportation cost from source $i$ to destination $\mathrm{j}$. Let $\tilde{\mathrm{X}}_{\mathrm{ij}}$ denote the number of units to be transported from source $\mathrm{i}$ to destination $\mathrm{j}$. Now the problem is to find a feasible way of transporting the available amount at each source to satisfy the demand at each destination so that the total interval transportation cost is minimized .

\section{Mathematical Formulation for using Interval Transportation Problem}

The mathematical model of fully interval transportation problem is as follows

$$
\begin{aligned}
& \text { Minimize } \tilde{Z} \approx \sum_{\mathrm{i}=1}^{m} \sum_{\mathrm{j}=1}^{\mathrm{n}} \tilde{\mathrm{c}}_{\mathrm{ij}} \tilde{\mathrm{x}}_{\mathrm{ij}} \\
& \text { subject to } \sum_{\mathrm{j}=1}^{\mathrm{n}} \tilde{\mathrm{x}}_{\mathrm{ij}} \approx \tilde{\mathrm{a}}_{\mathrm{i}}, \mathrm{i}=1,2,3, \ldots, \mathrm{m} \\
& \sum_{\mathrm{i}=1}^{\mathrm{m}} \tilde{\mathrm{x}}_{\mathrm{ij}} \approx \tilde{\mathrm{b}}_{\mathrm{j}}, \mathrm{j}=1,2,3, \ldots, \mathrm{n} \\
& \sum_{\mathrm{i}=1}^{\mathrm{m}} \tilde{\mathrm{a}}_{\mathrm{i}} \approx \sum_{\mathrm{j}=1}^{\mathrm{n}} \tilde{\mathrm{b}}_{\mathrm{j}} \text {, where } \mathrm{i}=1,2,3, \ldots, \mathrm{m} ; \mathrm{j}=1,2,3, \ldots, \mathrm{n} \\
& \text { and } \tilde{\mathrm{x}}_{\mathrm{ij}} \succeq \tilde{0} \text { for all } \mathrm{i} \text { and } \mathrm{j}
\end{aligned}
$$

And $\tilde{a}_{i}, \tilde{b}_{j}, \tilde{c}_{i j}, \tilde{x}_{i j}$ in IR, where $\tilde{c}_{i j}$ is the interval unit transportation cost from $i^{\text {th }}$ source to the $j^{\text {th }}$ destination . The goal is to minimize total fuzzy transportation costs, the problem of fuzzy transportation is solved in this paper by the max-min version interval method.

Definition: An interval feasible solution is defined as a set of non-negative assignments that satisfy (in the context corresponding) the row and the column boundaries .

Definition: An interval solution to a problem with $\mathrm{m}$ sources and $\mathrm{n}$ destinations in the transport interval is said to be a basic interval solution if the number of positive allocations is $(m+n-1)$. When the number allocations in a basic interval solution are less than $(m+n-1)$, then it is called the degenerate basic feasible solution .

Definition: An interval feasible solution is said to be optimal if it minimizes the total cost of transportation .

\section{General Form of Interval Transportation Problems with Multiple Objectives}

The mathematical formulation of interval transportation problems with multiple objectives when all the cost coefficient, supply and demand are interval numbers is given by: 


$$
\begin{aligned}
& \text { Minimize } \tilde{Z}^{\mathrm{k}} \approx \sum_{\mathrm{i}=1}^{\mathrm{m}} \sum_{\mathrm{j}=1}^{\mathrm{n}} \tilde{\mathrm{c}}_{\mathrm{ij}}^{\mathrm{k}} \tilde{\mathrm{x}}_{\mathrm{ij}}, \text { where } \mathrm{k}=1,2, \ldots, \mathrm{K} \\
& \text { subject to } \sum_{\mathrm{j}=1}^{\mathrm{n}} \tilde{\mathrm{x}}_{\mathrm{ij}} \approx \tilde{\mathrm{a}}_{\mathrm{i}}, \mathrm{i}=1,2,3, \ldots, \mathrm{m} \\
& \qquad \sum_{\mathrm{i}=1}^{\mathrm{m}} \tilde{\mathrm{x}}_{\mathrm{ij}} \approx \tilde{\mathrm{b}}_{\mathrm{j}}, \mathrm{j}=1,2,3, \ldots, \mathrm{n} \\
& \qquad \sum_{\mathrm{i}=1}^{\mathrm{m}} \tilde{\mathrm{a}}_{\mathrm{i}} \approx \sum_{\mathrm{j}=1}^{\mathrm{n}} \tilde{\mathrm{b}}_{\mathrm{j}}, \text { where } \mathrm{i}=1,2,3, \ldots, \mathrm{m} ; \mathrm{j}=1,2,3, \ldots, \mathrm{n}
\end{aligned}
$$

Where $\tilde{\mathrm{Z}}^{\mathrm{k}} \approx\left\{\tilde{\mathrm{Z}}^{1}, \tilde{\mathrm{Z}}^{2}, \ldots, \tilde{\mathrm{Z}}^{\mathrm{K}}\right\}$ is a vector of $\mathrm{K}$ objective functions and the superscript on both $\tilde{\mathrm{Z}}^{\mathrm{k}}$ and $\mathrm{c}_{\mathrm{ij}}^{\mathrm{k}}$ are used to identify the number of objective functions $(\mathrm{k}=1,2, \ldots, \mathrm{K})$.

\section{Weighted Sum (or) Scalarization Method}

By assigning suitable weights $\mathrm{w}_{\mathrm{k}}>0$ such that $\sum_{\mathrm{k}=1}^{\mathrm{K}} \mathrm{w}_{\mathrm{k}}=1$ to the $\mathrm{K}$ objective functions, the multiple objective interval transportation problem (4.2) becomes a single objective interval transportation problem

$$
\begin{aligned}
& \text { Minimize } \tilde{\mathbf{Z}} \approx \sum_{\mathrm{k}=1}^{\mathrm{K}} \mathrm{w}_{\mathrm{k}} \tilde{\mathrm{Z}}^{\mathrm{k}}, \text { where } \sum_{\mathrm{k}=1}^{\mathrm{K}} \mathrm{w}_{\mathrm{k}}=1 \text { and } \mathrm{w}_{\mathrm{k}}>0 . \\
& \text { subject to } \sum_{\mathrm{j}=1}^{\mathrm{n}} \tilde{\mathrm{x}}_{\mathrm{ij}} \approx \tilde{\mathrm{a}}_{\mathrm{i}}, \mathrm{i}=1,2,3, \ldots, \mathrm{m} \\
& \qquad \sum_{\mathrm{i}=1}^{\mathrm{m}} \tilde{\mathrm{x}}_{\mathrm{ij}} \approx \tilde{\mathrm{b}}_{\mathrm{j}}, \mathrm{j}=1,2,3, \ldots, \mathrm{n} \\
& \qquad \sum_{\mathrm{i}=1}^{\mathrm{m}} \tilde{\mathrm{a}}_{\mathrm{i}} \approx \sum_{\mathrm{j}=1}^{\mathrm{n}} \tilde{\mathrm{b}}_{\mathrm{j}}, \text { where } \mathrm{i}=1,2,3, \ldots, \mathrm{m} ; \mathrm{j}=1,2,3, \ldots, \mathrm{n}
\end{aligned}
$$

Definition: (Non-dominated solution). A feasible vector $\tilde{\mathbf{x}}^{*} \in \mathrm{X}$ (X is the feasible region) yields a non-dominated solution of (4.2) if and only if, there is no other feasible vector $\tilde{\mathbf{x}}^{*} \in \mathrm{X}$ such that $\sum_{\mathrm{i}=1}^{\mathrm{m}} \sum_{\mathrm{j}=1}^{\mathrm{n}} \tilde{\mathrm{i}}_{\mathrm{ij}}^{\mathrm{k}} \tilde{\mathrm{x}}_{\mathrm{ij}} \preceq \sum_{\mathrm{i}=1}^{\mathrm{m}} \sum_{\mathrm{j}=1}^{\mathrm{n}} \tilde{\mathrm{c}}_{\mathrm{ij}}^{\mathrm{k}} \tilde{\mathrm{x}}_{\mathrm{ij}}^{*}$, for all k and $\sum_{\mathrm{i}=1}^{\mathrm{m}} \sum_{\mathrm{j}=1}^{\mathrm{n}} \tilde{c}_{\mathrm{ij}}^{\mathrm{k}} \tilde{\mathrm{x}}_{\mathrm{ij}} \prec \sum_{\mathrm{i}=1}^{\mathrm{m}} \sum_{\mathrm{j}=1}^{\mathrm{n}} \tilde{\mathrm{c}}_{\mathrm{ij}}^{\mathrm{k}} \tilde{\mathrm{x}}_{\mathrm{ij}}^{*}$, for some $\mathrm{k}, \mathrm{k}=1,2, \cdots \mathrm{K}$.

Definition: (Efficient solution). A point $\tilde{\mathbf{x}}^{*} \in \mathrm{X}$ efficient iff there does not exist another $\tilde{\mathbf{x}} \in \mathrm{X}$ such that $\sum_{\mathrm{i}=1}^{m} \sum_{\mathrm{j}=1}^{\mathrm{n}} \tilde{c}_{\mathrm{ij}}^{\mathrm{k}} \tilde{x}_{\mathrm{ij}} \preceq \sum_{\mathrm{i}=1}^{\mathrm{m}} \sum_{\mathrm{j}=1}^{\mathrm{n}} \tilde{c}_{\mathrm{ij}}^{\mathrm{k}} \tilde{\mathrm{x}}_{\mathrm{ij}}^{*} \mathrm{all} \mathrm{k}$ and $\sum_{\mathrm{i}=1}^{\mathrm{m}} \sum_{\mathrm{j}=1}^{\mathrm{n}} \tilde{c}_{\mathrm{ij}}^{\mathrm{k}} \tilde{\mathrm{x}}_{\mathrm{ij}} \neq \sum_{\mathrm{i}=1}^{\mathrm{m}} \sum_{\mathrm{j}=1}^{\mathrm{n}} \tilde{c}_{\mathrm{ij}}^{\mathrm{k}} \tilde{\mathrm{x}}_{\mathrm{ij}}^{*}$, for some $\mathrm{k}$.

Definition: (Compromise solution). A feasible vector $\tilde{\mathbf{x}}^{*} \in \mathrm{X}$ is called a compromise solution of (4.2) if and only if $\tilde{\mathbf{x}}^{*} \in \operatorname{IR}^{\mathrm{n}}$ and $\tilde{\mathbf{Z}}\left(\tilde{\mathbf{x}}^{*}\right) \wedge_{\tilde{\mathbf{x}} \in \mathrm{X}} \tilde{\mathbf{Z}}(\tilde{\mathbf{x}})$, where $\wedge$ stands for minimum and $\mathrm{X}$ is the set of efficient solutions.

Theorem: If $\tilde{\mathbf{x}}^{*} \in \mathrm{X}$ is an optimum solution the single objective interval transportation problem (4.3), then it is also a compromised (pareto optimal) solution to the multi-objective interval transportation problem (4.2) 


\section{INTERVAL MAX-MIN ALGORITHM FOR INITIAL BASIC FEASIBLE SOLUTION}

Step 1: Present the problem of multi-objective interval transportation in the transport table.

Step 2: Examine if the total supply is equal to the total demand, then it is a balanced problem, If not, add dummy row / column with zero cost and time.

Step 3: Express the all interval parameters supply, demand and unit transportation cost, time etc in the Multiobjective transportation problem in terms of midpoint and half width. That is in the form of $\tilde{a}=\left[a_{1}, a_{2}\right]=\langle m(\tilde{a}), w(\tilde{a})\rangle$.

Step 4: Construct the single objective interval transportation problem (4.3) by giving suitable weights $\mathrm{w}_{\mathrm{k}}>0$ such that $\sum_{\mathrm{k}=1}^{\mathrm{K}} \mathrm{w}_{\mathrm{k}}=1$ to the $\mathrm{K}$ objective functions

Step 5: Compute the row-wise difference between maximum and minimum (Penalty) of each row, and it is divided by the number of columns of the cost matrix.

Step 6: Compute the column-wise difference between maximum and minimum (Penalty) of each column, and it is divided by the number of rows of the cost matrix.

Step 7: Select a row or column with the maximum of the penalty and identify the corresponding minimum cost cell and do the allocation of that particular cell of the given matrix. It there is a tie, break the tie arbitrarily.

Step 8: Repeated procedures 1 to 7 until all the allocations are completed

Note: Check the optimality of the initial basic feasible solution obtained using the interval version of the MODI method.

\section{NUMERICAL RESULTS}

Consider Ummey Habiba and Abdul Quddoos' following multi objective interval transport problem [20]

Table 1: Interval Transportation Cost

\begin{tabular}{|c|c|c|c|c|c|}
\hline & D1 & D2 & D3 & D4 & Supply \\
\hline S1 & {$[10,12]$} & {$[15,16]$} & {$[21,24]$} & {$[21,25]$} & 967.54 \\
\hline S2 & {$[15,25]$} & {$[10,20]$} & {$[9,11]$} & {$[18,19]$} & 762.93 \\
\hline S3 & {$[20,26]$} & {$[10,17]$} & {$[20,25]$} & {$[15,20]$} & 612.82 \\
\hline Demand & 615.99 & 511.88 & 408.35 & 305.25 & \\
\hline
\end{tabular}

Table 2: Interval Transportation Time

\begin{tabular}{|c|c|c|c|c|c|}
\hline & D1 & D2 & D3 & D4 & Supply \\
\hline S1 & {$[9,11]$} & {$[16,17]$} & {$[21,24]$} & {$[16,18]$} & 967.54 \\
\hline S2 & {$[9,13]$} & {$[14,19]$} & {$[16,18]$} & {$[19,20]$} & 762.93 \\
\hline S3 & {$[9,17]$} & {$[20,26]$} & {$[25,27]$} & {$[28,29]$} & 612.82 \\
\hline Demand & 615.99 & 511.88 & 408.35 & 305.25 & \\
\hline
\end{tabular}

Since $\sum_{\mathrm{i}=1}^{\mathrm{m}} \tilde{\mathrm{a}}_{\mathrm{i}} \neq \sum_{\mathrm{j}=1}^{\mathrm{n}} \tilde{\mathrm{b}}_{\mathrm{j}}$, the given interval transportation problem with multiple objectives is unbalanced.

By introducing a dummy destination $\mathrm{D}_{5}$ with zero cost and time, the given multi-objective interval transportation problem becomes balanced. 
Express all the interval parameters in terms of their midpoint, width form, we have

Table 3: Interval Transportation Cost in Mid Point and Width Form

\begin{tabular}{|c|c|c|c|c|c|c|}
\hline & D1 & D2 & D3 & D4 & D5 & Supply \\
\hline S1 & $\langle 11,1\rangle$ & $\langle 15.5,0.5\rangle$ & $\langle 22.5,1.5\rangle$ & $\langle 23,2\rangle$ & $\langle 0,0\rangle$ & $\langle 967.54,0\rangle$ \\
\hline S2 & $\langle 20,5\rangle$ & $\langle 15,5\rangle$ & $\langle 10,1\rangle$ & $\langle 18.5,0.5\rangle$ & $\langle 0,0\rangle$ & $\langle 762.93,0\rangle$ \\
\hline S3 & $\langle 23,3\rangle$ & $\langle 13.5,3.5\rangle$ & $\langle 22.5,2.5\rangle$ & $\langle 17.5,2.5\rangle$ & $\langle 0,0\rangle$ & $\langle 612.82\rangle$ \\
\hline Demand & $\langle 615.99,0\rangle$ & $\langle 511.88,0\rangle$ & $\langle 408.35,0\rangle$ & $\langle 305.25,0\rangle$ & $\langle 501.82,0\rangle$ & $\langle 2343.29\rangle$ \\
\hline
\end{tabular}

Table 4: F. Interval Transportation Time in Mid Point and Width Form

\begin{tabular}{|c|c|c|c|c|c|c|}
\hline & D1 & D2 & D3 & D4 & D5 & Supply \\
\hline S1 & $\langle 10,1\rangle$ & $\langle 16.5,0.5\rangle$ & $\langle 22.5,1.5\rangle$ & $\langle 17,1\rangle$ & $\langle 0,0\rangle$ & $\langle 967.54,0\rangle$ \\
\hline S2 & $\langle 11,2\rangle$ & $\langle 16.5,2.5\rangle$ & $\langle 17,1\rangle$ & $\langle 19.5,0.5\rangle$ & $\langle 0,0\rangle$ & $\langle 762.93,0\rangle$ \\
\hline S3 & $\langle 13,4\rangle$ & $\langle 23,3\rangle$ & $\langle 26,1\rangle$ & $\langle 28.5,0.5\rangle$ & $\langle 0,0\rangle$ & $\langle 612.82\rangle$ \\
\hline Demand & $\langle 615.99,0\rangle$ & $\langle 511.88,0\rangle$ & $\langle 408.35,0\rangle$ & $\langle 305.25,0\rangle$ & $\langle 501.82,0\rangle$ & $\langle 2343.29\rangle$ \\
\hline
\end{tabular}

By assigning suitable weights $\mathrm{w}_{1}=\mathrm{r}, \mathrm{w}_{2}=(1-\mathrm{r})$, respectively to the cost and time interval parameters and combining them, single objective interval transportation problem becomes.

Table 5: Single Objective Interval Transportation Problem

\begin{tabular}{|c|c|c|c|c|c|c|}
\hline & D1 & D2 & D3 & D4 & D5 & Supply \\
\hline S1 & $\langle 10+\mathrm{r}, 1\rangle$ & $\langle 16.5-\mathrm{r}, 0.5\rangle$ & $\langle 22.5+0 \mathrm{r}, 1.5\rangle$ & $\langle 17+6 \mathrm{r}, 1\rangle$ & $\langle 0+0 \mathrm{r}, 0\rangle$ & $\langle 967.54,0\rangle$ \\
\hline S2 & $\langle 11+9 \mathrm{r}, 5\rangle$ & $\langle 16.5-1.5 \mathrm{r}, 5\rangle$ & $\langle 17-7 \mathrm{r}, 1\rangle$ & $\langle 19.5-\mathrm{r}, 0.5\rangle$ & $\langle 0+0 \mathrm{r}, 0\rangle$ & $\langle 762.93,0\rangle$ \\
\hline S3 & $\langle 13+10 \mathrm{r}, 4\rangle$ & $\langle 23-9.5 \mathrm{r}, 3.5\rangle$ & $\langle 26-3.5 \mathrm{r}, 2.5\rangle$ & $\langle 28.5-11 \mathrm{r}, 2.5\rangle$ & $\langle 0+0 \mathrm{r}, 0\rangle$ & $\langle 612.82\rangle$ \\
\hline Demand & $\langle 615.99,0\rangle$ & $\langle 511.88,0\rangle$ & $\langle 408.35,0\rangle$ & $\langle 305.25,0\rangle$ & $\langle 501.82,0\rangle$ & $\langle 2343.29\rangle$ \\
\hline
\end{tabular}

By applying the proposed algorithm, the initial basic feasible solution is obtained as

Table 6: Initial Basic Feasible Solution

\begin{tabular}{|c|c|c|c|c|c|c|}
\hline & D1 & D2 & D3 & D4 & D5 & Supply \\
\hline $\mathrm{S} 1$ & $\begin{array}{c}\langle 10+\mathrm{r}, 1\rangle \\
\langle 504.99,0\rangle\end{array}$ & $\begin{array}{c}<16.5-\mathrm{r}, 0.5> \\
<157.3,0>\end{array}$ & $<22.5+0 \mathrm{r}, 1.5\rangle$ & $\begin{array}{l}<17+6 r, 1> \\
<305.25,0>\end{array}$ & $<0+0 r, 0>$ & $\langle 967.54,0\rangle$ \\
\hline $\mathrm{S} 2$ & $<11+9 r, 5>$ & $\begin{array}{c}\langle 16.5-1.5 \mathrm{r}, 5\rangle \\
<354.58,0\rangle\end{array}$ & $\begin{array}{c}<17-7 \mathrm{r}, 1> \\
<408.35,0>\end{array}$ & $<19.5-r, 0.5>$ & $<0+0 r, 0>$ & $\langle 762.93,0\rangle$ \\
\hline $\mathrm{S} 3$ & $\begin{array}{c}<13+10 \mathrm{r}, 4> \\
<111,0>\end{array}$ & $\langle 23-9.5 r, 3.5\rangle$ & $\langle 26-3.5 r, 2.5\rangle$ & $\langle 28.5-11 \mathrm{r}, 2.5\rangle$ & $\begin{array}{c}\langle 0+0 \mathrm{r}, 0\rangle \\
\langle 501.82,0\rangle\end{array}$ & $<612.82>$ \\
\hline Demand & $\langle 615.99,0\rangle$ & $\langle 511.88,0\rangle$ & $\langle 408.35,0\rangle$ & $\langle 305.25,0\rangle$ & $\langle 501.82,0\rangle$ & $\langle 2343.29\rangle$ \\
\hline
\end{tabular}

Since the number of positive allocations is $(m+n-1)=7$, interval version of the MODI method is applied to check the optimality of the current solution and is found to be optimal. Now by theorem (4.1), this optimal solution will be a pareto optimal solution to the given multi objective interval transport problem.

Hence the compromised solution to the given multi objective interval transport problem is

$$
\begin{aligned}
& \mathrm{x}_{11}=\langle 504.99,0\rangle=504.99, \mathrm{x}_{12}=\langle 157.3,0\rangle=157.3, \mathrm{x}_{14}=\langle 305.25,0\rangle=305.25, \\
& \mathrm{x}_{22}=\langle 354.58,0\rangle=354.58, \mathrm{x}_{23}=\langle 408.35\rangle=408.35, \mathrm{x}_{31}=\langle 111,0\rangle=111, \mathrm{x}_{35}=\langle 501.82,0\rangle=501.82
\end{aligned}
$$

The corresponding minimum cost $\left.\left.\tilde{Z}^{1}=\langle 11,1\rangle\langle 504.99,0\rangle+<15.5,0.5\right\rangle\langle 157.3,0\rangle+<23,2\right\rangle\langle 305.25,0\rangle$

$$
\begin{aligned}
& +<15,5\rangle\langle 354.58,0\rangle+\langle 10,1\rangle\langle 408.35,0\rangle+\langle 23,3\rangle\langle 111,0\rangle \\
& +<0,0\rangle\langle 501.82,0\rangle \\
& =<26968.99,5\rangle
\end{aligned}
$$




$$
=[26963.99,26973.99] .
$$

The corresponding minimum time $\tilde{Z}^{2}=\langle 10,1\rangle\langle 504.99,0\rangle+\langle 16.5,0.5\rangle\langle 157.3,0\rangle+\langle 17,1\rangle\langle 305.25,0\rangle$

$$
\begin{aligned}
& +\langle 16.5,2.5\rangle\langle 354.58,0\rangle+\langle 17,1\rangle\langle 408.35,0\rangle+\langle 13,4\rangle\langle 111,0\rangle \\
& +<0,0\rangle\langle 501.82,0\rangle \\
& =\langle 27070.12,2.5\rangle \\
& =[27067.62,27072.62]
\end{aligned}
$$

Table 7: Comparison of Solutions

\begin{tabular}{|c|c|c|}
\hline S. No. & Solution by the proposed method & Solution by Ummey Habiba and Abdul Quddoos' [20] \\
\hline 1 & $Z^{1}=[26963.99,26973.99]$ & $Z^{1}=[21975.32,27265.83]$ \\
\hline 2 & $Z^{2}=[27067.62,27072.62]$ & $Z^{2}=[25841.42,30004.25]$ \\
\hline
\end{tabular}

It is seen from the table that the solution obtained by the proposed method gives vagueness reduced pareto optimal values for both the objective functions

\section{CONCLUSIONS}

In this paper, we suggested interval variants of the Max-Min approach to address multi-objective interval transportation problems without translating them to traditional multi-objective transportation problem. From a practical point of view, the Max-Min process approach is very simple and easy to understand and to apply. The approach presented and discussed above gives us an initial feasible simple solution, which is closer to the optimal solution of the problem of multi-objective interval transport. It also provides vagueness reduced pareto optimal values for both the objective functions

\section{REFERENCES}

1. Akilbasha, P.Pandian and G.Natarajan, An innovative exact method for solving fully interval integer transportation problems, Informatics in Medicine Unlocked, 11, (2018), 95-99.

2. Chanas S., Delgado M., Verdegay J.L, Vila M.A., Interval and fuzzy extensions of classical transportation problems, Transportation Planning Technology, 17, 1993.

3. J.W. Chinneck and K. Ramadan, Linear programming with interval coefficients, Journal of the Operational Research Society, 51, (2000), $209-220$.

4. S. K. Das, A. Goswami and S.S. Alam, Multiobjective transportation problem with interval cost, source and destination parameters, European Journal of Operational Research, 117 (1999), 100 - 112.

5. Raj, Sona, and Vikas H. Pradhan. "Numerical Simulation Of One-Dimensional Solute Transport Equation By Using Differential Quadrature Method." International Journal of Mathematics and Computer Applications Research, Tran stellar 3.3 (2013).

6. M. R. Fegade, V. A. Jadhav and A. A. Muley, Finding an optimal solution of transportation problem using interval and triangular membership functions, European Journal of Scientific Research, 60 (3) (2011),397- 403.

7. K. Ganesan and P. Veeramani, On Arithmetic Operations of Interval Numbers, International Journal of Uncertainty, Fuzziness and Knowledge - Based Systems, 13 (6) (2005), 619 - 631. 
8. K. Ganesan, On Some Properties of Interval Matrices, International Journal of Computational and Mathematical Sciences, 1 (2) (2007), 92 - 99.

9. Gurupada Maity, Sankar Kumar Roy and Jose Luis Verdegay, Time variant multi-objective interval-valued transportation problem in sustainable development, sustainability, 11, (2019), 01-15.

10. Chitte, Pritish, et al. "Design and Fabrication of Automated Pneumatic Shearing Machine to Cut Aluminium Sheet"." International Journal of Mechanical and Production Engineering Research and Development, p-ISSN (2018): 22496890 (2018).

11. Ishibuchi, H., Tanaka, H., Multi objective programming in optimization of the interval objective function. European Journal of Operational Research, 48 (1990), 219-225.

12. Ming Ma, Menahem Friedman and Abraham Kandel. 1999. A new fuzzy arithmetic. Fuzzy sets and systems. 108: 8390.

13. C. Oliveira and C.H. Antunes, Multiple objective linear programming models with interval coefficients - an illustrated overview, European Journal of Operational Research, 181, (2007), 1434-1463.

14. P. Pandian and G. Natrajan, A new method for finding an optimal solution of fully interval integer transportation problems, applied mathematical sciences. Applied 4(37) (2010), 1819 - 1830.

15. Putra, Adris Ade, Edward Ngii, and Susanti Djalante. "Port Development In Supporting Connectivity System Of Archipelago Region." International Journal of Mechanical and Production Engineering Research and Development (IJMPERD) 8 (2018).

16. G. Ramesh and K. Ganesan, Interval Linear Programming with generalized interval arithmetic, International Journal of Scientific \& Engineering Research, 2 (11), November-2011.

17. G. Ramesh and K. Ganesan, Duality theory for interval linear programming problems, IOSR Journal of Mathematics (IOSRJM), 4 (4) (2012), 39 - 47.

18. G. Ramesh, G.Sudha and K.Ganesan, Solution of two vehicle cost varying interval transportation problem-a new approach, International Journal of Pure and Applied Mathematics, 119(9), (2018), 363-372

19. Madura, Vijaya Kumar, et al. "Design and experimental study of semi active system of MR damper for vibration control." International Journal of Mechanical and Production Engineering Research and Development 8.5 (2018): $125-132$.

20. Safi, M. R., and A. Razmjoo, Solving Fixed Charge Transportation Problem with Interval Parameters, Applied Mathematical Modelling, 37 (18-19), (2013), 8341-8347.

21. A. Sengupta and T.K. Pal, Interval-valued transportation problem with multiple penalty factors, VU Journal of Physical Sciences, 9, (2003), $71-81$.

22. Sophia Porchelvi and M. Anitha, Comparative study of optimum solution between interval transportation and interval transhipment problem, International Journal of Advanced Science and Engineering, 4 (4), (2018), 764-767.

23. S. Sathya Geethal, K. Selvakumari, A New method for solving fuzzy Transportation problem using pentagonal fuzzy numbers. Journal of Critical Reviews, Vol 7, Issue 9, (2020), ISSN- 2394-5125

24. Ummey Habiba and Abdul Quddoos. Multiobjective Stochastic Interval Transportation Problem Invoiving general form of distributions, Advances in Mathematics: Scientific Journal 9 (2020), no.6, 3213-3219 ISSN: 1857-8365 (printed); 1857-8438 (electronic) 
25. V.J. Sudhakar and V. Navaneetha Kumar, A New Approach for Finding an Optimal Solution for Integer Interval Transportation Problems, Int. J. Open Problems Compt. Math., 3 (5), (2010), 131-137. 